\title{
ON A LIFT OF AN INDIVIDUAL STABLE EQUIVALENCE TO A STANDARD DERIVED EQUIVALENCE FOR REPRESENTATION-FINITE SELFINJECTIVE ALGEBRAS
}

\author{
HIDETO ASASHIBA \\ Dedicated to Professor Idun Reiten on the occasion of her 60-th birthday
}

\begin{abstract}
We shall show that every stable equivalence (functor) between representation-finite selfinjective algebras not of type $\left(D_{3 m}, s / 3,1\right)$ with $m \geq 2,3 \nmid s$ lifts to a standard derived equivalence. This implies that all stable equivalences between these algebras are of Morita type.
\end{abstract}

\section{INTRODUCTION}

Throughout this paper $k$ denotes an algebraically closed field, and unless otherwise stated, all algebras considered here are assumed to be basic, connected, finitedimensional $k$-algebras with identity. For an algebra $\Lambda$, we denote by $\bmod \Lambda$, by pro $\Lambda$ and by $\underline{\bmod } \Lambda$ the category of finite-dimensional (right) $\Lambda$-modules, the full subcategory of $\bmod \Lambda$ consisting of finitely generated projective $\Lambda$-modules and the stable category of $\bmod \Lambda$, respectively. Further by $\mathcal{D}^{\mathrm{b}}(\bmod \Lambda)$ and by $\mathcal{H}^{\mathrm{b}}(\operatorname{pro} \Lambda)$ the bounded derived category of $\bmod \Lambda$ and the bounded homotopy category of pro $\Lambda$, respectively. Recall that $\underline{\bmod } \Lambda$ is the factor category $(\bmod \Lambda) /\langle\operatorname{pro} \Lambda\rangle$, where $\langle\operatorname{pro} \Lambda\rangle$ is the ideal of $\bmod \Lambda$ consisting of morphisms of $\bmod \Lambda$ factoring through an object in pro $\Lambda$. Then we have the canonical functor $\pi_{\Lambda}: \bmod \Lambda \rightarrow \underline{\bmod } \Lambda$. When $\Lambda$ is a selfinjective algebra, the category $\underline{\bmod } \Lambda$ is naturally regarded as a triangulated category.

For two algebras $\Lambda$ and $\Pi$, an equivalence $\underline{\bmod } \Lambda \rightarrow \underline{\bmod } \Pi$ is called a stable equivalence from $\Lambda$ to $\Pi$. The algebras $\Lambda$ and $\Pi$ are said to be stably equivalent if there exists a stable equivalence between them. Following Broué [5] (see also Linckelmann [7]) a stable equivalence $\phi: \underline{\bmod } \Lambda \rightarrow \underline{\bmod } \Pi$ is called of Morita type if there exist bimodules ${ }_{\Lambda} M_{\Pi,}{ }_{\Pi} N_{\Lambda}$ such that the following three conditions are satisfied:

(1) ${ }_{\Lambda} M, M_{\Pi},{ }_{\Pi} N$ and $N_{\Lambda}$ are projective modules;

(2) (a) $M \otimes_{\Pi} N \cong \Lambda \oplus P$ in $\bmod \Lambda^{\mathrm{op}} \otimes_{k} \Lambda$ for some $P \in$ pro $\Lambda^{\mathrm{op}} \otimes_{k} \Lambda$, and

(b) $N \otimes_{\Lambda} M \cong \Pi \oplus Q$ in $\bmod \Pi^{\mathrm{op}} \otimes_{k} \Pi$ for some $Q \in$ pro $\Pi^{\mathrm{op}} \otimes_{k} \Pi$; and

(3) $\phi$ lifts to the functor $-\otimes_{\Lambda} M$, i.e., the diagram

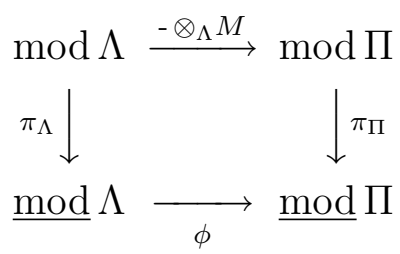

commutes up to natural isomorphisms. 
Note that the $\phi$ above lifts to a Morita equivalence if and only if it is of Morita type with $P=0=Q$ in the definition. An example of a stable equivalence of Morita type is given by the loop functor over a selfinjective algebra, as we shall see later. In particular, the loop functor over a (non-semisimple) selfinjective Nakayama algebra gives an example of a stable equivalence of Morita type that does not lift to a Morita equivalence.

On the other hand, for two algebras $\Lambda$ and $\Pi$, a triangle equivalence $\Phi: \mathcal{D}^{\mathrm{b}}(\bmod \Lambda) \rightarrow$ $\mathcal{D}^{\mathrm{b}}(\bmod \Pi)$ is called a derived equivalence from $\Lambda$ to $\Pi$. The algebras $\Lambda$ and $\Pi$ are said to be derived equivalent if there exists a derived equivalence between them. If $\Phi$ has the form $\Phi=-\otimes_{\Lambda}^{\mathbf{L}} M^{\bullet}$ for some twosided tilting complex $M^{\bullet}$ of $\Lambda$ - $\Pi$-bimodules, then $\Phi$ is called a standard derived equivalence. When $\Lambda$ is selfinjective it has been shown in [6] or in [9] that the embedding functor $\bmod \Lambda \rightarrow \mathcal{D}^{\mathrm{b}}(\bmod \Lambda)$ yields an equivalence $\sigma_{\Lambda}: \underline{\bmod } \Lambda \rightarrow \mathcal{D}^{\mathrm{b}}(\bmod \Lambda) / \mathcal{H}^{\mathrm{b}}(\operatorname{pro} \Lambda)$ of triangulated categories. Let $\sigma_{\Lambda}^{-1}$ be the quasiinverse of $\sigma_{\Lambda}$ given in [9]. Denote the composite of the functors

$$
\mathcal{D}^{\mathrm{b}}(\bmod \Lambda) \stackrel{\text { can }}{\longrightarrow} \mathcal{D}^{\mathrm{b}}(\bmod \Lambda) / \mathcal{H}^{\mathrm{b}}(\operatorname{pro} \Lambda) \stackrel{\sigma_{\Lambda}^{-1}}{\longrightarrow} \underline{\bmod } \Lambda
$$

by $\eta_{\Lambda}: \mathcal{D}^{\mathrm{b}}(\bmod \Lambda) \rightarrow \underline{\bmod } \Lambda$.

A stable equivalence $\phi: \underline{\bmod } \Lambda \rightarrow \underline{\bmod } \Pi$ of selfinjective algebras $\Lambda$ and $\Pi$ is said to lift to a derived equivalence $\Phi: \mathcal{D}^{\mathrm{b}}(\overline{\bmod } \Lambda) \rightarrow \mathcal{D}^{\mathrm{b}}(\bmod \Pi)$ in case the diagram

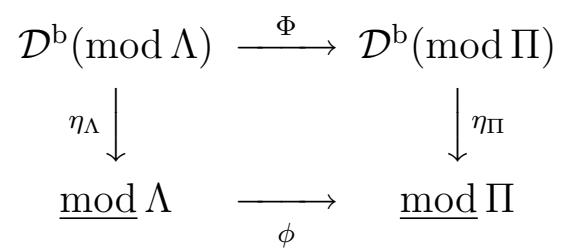

commutes up to natural isomorphisms. Note that if $\phi$ above lifts to a Morita equivalence, then $\phi$ lifts to a standard derived equivalence.

In [10] Rickard has shown that a standard derived equivalence of selfinjective algebras supplies a stable equivalence of Morita type. We shall see later (in section 5) that if a stable equivalence $\phi$ of selfinjective algebras lifts to a standard derived equivalence $\Phi$, then the stable equivalence of Morita type obtained from $\Phi$ coincides with $\phi$. This is not clear from the proof given in [10, Corollary 5.5], and means that a stable equivalence of selfinjective algebras liftable to a standard derived equivalence is of Morita type.

Since the condition to be of Morita type is rather strong, most stable equivalences seem to be not of Morita type. But in constructing stable equivalences one usually uses those of Morita type. Therefore it seems that only few examples of stable equivalences not of Morita type are known. In looking for such examples in the class of representation-finite selfinjective algebras, we realized that by contraries it is impossible to find them in most cases in this class. Further the argument used in this proof could be easily extended to obtain the following.

Main Theorem. Let $\Lambda$ and $\Pi$ be selfinjective algebras. Assume that $\Lambda$ is representationfinite and not of type $\left(D_{3 m}, s / 3,1\right)$ with $m \geq 2,3 \nmid s$. Then every stable equivalence between $\Lambda$ and $\Pi$ lifts to a standard derived equivalence, and hence in particular, it is of Morita type. 
See the next section for the definition of types of representation-finite selfinjective algebras. At present we do not know whether there exist stable equivalences that do not lift to standard derived equivalences in the remaining cases. However, Proposition 3.3 suggests us that at least in the remaining standard cases all stable equivalences would be of Morita type.

In section 1, we recall the derived equivalence classification of representation-finite (standard) selfinjective algebras from [1]. In section 2, we investigate the stability of configurations of the universal coverings $\tilde{\Lambda}$ of representative algebras $\Lambda$ in the derived equivalence classification (in section 1) under "essential" automorphisms of the stable Auslander-Reiten quivers of $\tilde{\Lambda}$. In section 3 , using the result in section 2 we describe the group $\operatorname{StPic}(\Lambda)$ of natural isomorphism classes of stable self-equivalences of $\Lambda$ for each representative algebra $\Lambda$. In particular, it is shown that $\operatorname{StPic}(\Lambda)$ is generated by the loop functor and the ones liftable to Morita equivalences for each representative algebra $\Lambda$ the type of which is treated in Main Theorem. In section 4 we notice that the loop functor lifts to a standard derived equivalence, and we give a proof of Main Theorem by reducing the problem to stable self-equivalences of representative algebras. Section 5 is an appendix in which for the benefit of the reader we recall the construction of $\eta_{\Lambda}$, and prove a proposition that produces both the construction of a stable equivalence of Morita type from a standard derived equivalence ([10, Corollary 5.5]) and a proof of the fact that a stable equivalence liftable to a standard derived equivalence is of Morita type. Finally section 6 is also an appendix in which we give explicit quiver presentations of all the representative algebras, which were presented by now as quotients of repetitions of tilted algebras (most of which were hereditary algebras) in $[1,2.5]$. We hope that this will help to study invariants of representationfinite selfinjective algebras under derived equivalences.

\section{Preliminaries}

Note that Main Theorem holds trivially if $\Lambda$ is semisimple. Therefore throughout the rest of the paper we assume that all selfinjective algebras considered here are not semisimple, or equivalently that they are not isomorphic to the one-dimensional algebra $k$. By $D, \Omega_{\Lambda}$ and $\tau_{\Lambda}$ we denote the usual $k$-dual functor $\operatorname{Hom}_{k}(-, k)$, the loop functor and the Auslander-Reiten translation of an algebra $\Lambda$, respectively. For algebras $R$ and $S$ over a commutative ring $K$, we regard $R$-S-bimodules as right $R^{\mathrm{op}} \otimes_{K} S$-modules in an obvious way.

Recall from [1] the definition of the type $\operatorname{typ}(\Lambda)$ of a representation-finite selfinjective algebra $\Lambda$. By Riedtmann [11] it is known that the stable Auslander-Reiten quiver of $\Lambda$ has the form $\mathbb{Z} \Delta /\langle g\rangle$, where $\Delta$ is a Dynkin graph, $r$ is a natural number, $g=\zeta \tau^{-r}$ and $\zeta$ is an automorphism of the quiver $\mathbb{Z} \Delta$ with a fixed vertex. In this case, we set $\operatorname{typ}(\Lambda):=(\Delta, f, t)$, where $f:=r / m_{\Delta}$ and $t$ is the order of $\zeta$. Here $m_{\Delta}$ is equal to $n$, $2 n-3,11,17$ or 29 according as $\Delta=A_{n}, D_{n}, E_{6}, E_{7}$ or $E_{8}$, respectively. Note that if $n$ is the number of vertices of $\Delta$, then $n f$ is equal to the number of isoclasses of simple $\Lambda$-modules (Bretscher et al. [4]). Thus we have $f=s / n$. We recall the following.

Proposition $1.1([1])$. The set of all the types of representation-finite selfinjective algebras $(\mp k)$ is equal to the disjoint union of the following sets: 


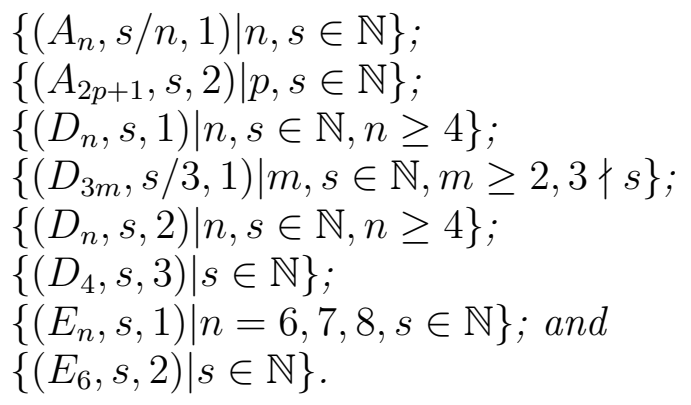

Notice that non-standard algebras have type $\left(D_{3 m}, 1 / 3,1\right)$ for some $m \geq 2$. Hence all the algebras in Main Theorem are standard algebras.

Recall that algebras $\Lambda$ and $\Pi$ are said to be derived equivalent (Rickard [8]) if the bounded derived categories of $\bmod \Lambda$ and $\bmod \Pi$ are equivalent as triangulated categories. If this is the case, then there exists a standard derived equivalence between $\Lambda$ and $\Pi$, which supplies a stable equivalence between $\Lambda$ and $\Pi$ of Morita type by Rickard [10]. With this in mind we have the following:

Theorem $1.2([1])$. Let $\Lambda$ and $\Pi$ be representation-finite standard selfinjective algebras. Then the following are equivalent:

(1) There exists a standard derived equivalence between $\Lambda$ and $\Pi$;

(2) $\Lambda$ and $\Pi$ are derived equivalent;

(3) There exists a stable equivalence of Morita type between $\Lambda$ and $\Pi$;

(4) $\Lambda$ and $\Pi$ are stably equivalent; and

(5) $\operatorname{typ}(\Lambda)=\operatorname{typ}(\Pi)$.

For each type $(\Delta, f, t)$ we gave a representative algebra $\Lambda(\Delta, f, t)$ that is a representation-finite standard selfinjective algebra of type $(\Delta, f, t)$ as quotients of repetitions of tilted algebras (see $[1,2.4$ and 2.5$])$. We set $\operatorname{can}(\Lambda):=\Lambda(\operatorname{typ}(\Lambda))$ for each representation-finite standard selfinjective algebra $\Lambda$. See Appendix 2 for the quiver presentations of all the representatives.

\section{Stability of CONFigurations}

For $\Delta=A_{n}(n \geq 1), D_{n}(n \geq 4), E_{6}$, we define an automorphism $\eta$ of $\mathbb{Z} \Delta$ and for $\Delta=D_{4}$ we define an automorphism $\eta^{\prime}$ of $\mathbb{Z} \Delta$ below to give a description of all automorphisms of $\mathbb{Z} \Delta$.

If $\Delta=A_{n}$ with $n$ odd, say $n=2 p+1$, then let $\vec{\Delta}$ be the quiver $\overrightarrow{A_{p}^{p}}$ :

$$
1 \leftarrow 2<\cdots<p<(p+1) \longrightarrow(p+2) \longrightarrow \cdots \longrightarrow n
$$

and define $\rho_{\Delta}$ by $x \mapsto n+1-x$.

If $\Delta=A_{n}$ with $n$ even, then let $\vec{\Delta}$ be the quiver $\overrightarrow{A_{n}}$ :

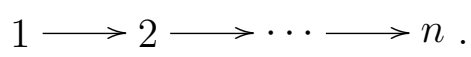


If $\Delta=D_{n}$, then let $\vec{\Delta}$ be the quiver $\overrightarrow{D_{n}}$ :

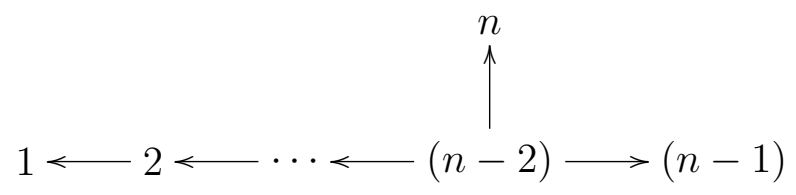

and define $\rho_{\Delta}$ by the permutation $(n, n-1)$. When $n=4$, define $\rho_{1}, \rho_{2}, \rho_{3}, \rho_{4}, \rho_{5}$ by the permutations $(1,3),(1,4),(3,4),(1,3,4),(1,4,3)$, respectively.

If $\Delta=E_{6}$, then let $\vec{\Delta}$ be the quiver $\vec{E}_{6}$ :

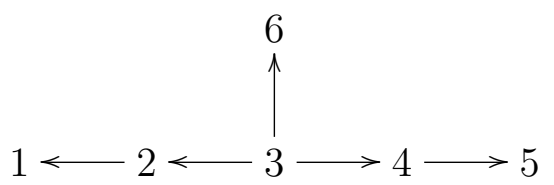

and define $\rho_{\Delta}$ by the permutation $(1,5)(2,4)$.

We introduce the coordinate of $\mathbb{Z} \Delta$ by identifying as $\mathbb{Z} \Delta=\mathbb{Z} \vec{\Delta}$. Then any automorphism $\rho$ of $\vec{\Delta}$ induces an automorphism $\mathbb{Z} \rho$ of $\mathbb{Z} \Delta$ by defining $(\mathbb{Z} \rho)(i, x):=(i, \rho(x))$ for all $i \in \mathbb{Z}$ and for all vertices $x$ of $\vec{\Delta}$.

Now define $\eta$ as follows. If $\Delta=A_{n}$ with $n$ odd, $\Delta=D_{n}$ or $\Delta=E_{6}$, then define $\eta:=\mathbb{Z} \rho_{\Delta}$. If $\Delta=A_{n}$ with $n$ even, say $n=2 p$, then define $\eta$ by $\eta(i, x):=(i+x-p-$ $1, n+1-x)$; in this case $\eta$ satisfies $\tau=\eta^{2}$. For $\Delta=D_{4}$, define $\eta_{i}:=\mathbb{Z} \rho_{i}$ for $i=1,2,3,4$ and 5. With these definitions we have the following that is due to Riedtmann [11].

Proposition 2.1. Let $\Delta=A_{n}(n \geq 1), D_{n}(n \geq 4)$ or $E_{n}(n=6,7,8)$. Then any automorphism of $\mathbb{Z} \Delta$ has the form $\tau^{a} \rho$ for some $\rho \in\left\{\mathbb{1}, \eta, \eta_{1}, \eta_{2}, \eta_{3}, \eta_{4}, \eta_{5}\right\}$ and some $a \in \mathbb{Z}$.

Now let $\Lambda$ be one of the representative algebras, thus $\Lambda=\operatorname{can}(\Lambda)$. Set $\operatorname{typ}(\Lambda)=$ $(\Delta, f, t)$. Then the Auslander-Reiten quiver of $\Lambda$ has the form $(\mathbb{Z} \Delta)_{\mathcal{C}} /\langle g\rangle$ for some configuration $\mathcal{C}$ of $\mathbb{Z} \Delta$, where $g=\zeta \tau^{-f m_{\Delta}}$ and $\zeta$ is an automorphism of $\mathbb{Z} \Delta$ with a fixed vertex and with order $t$. It is easy to calculate the $\mathcal{C}$ (e.g. use the argument in $[4,4.1]$ and the form of $B$ in $[1,2.5])$. We collect necessary information on $\mathcal{C}$ below.

If $\operatorname{typ}(\Lambda)=\left(A_{n}, s / n, 1\right)$, then $\mathcal{C}=\{(i, n) \mid i \in \mathbb{Z}\}$.

If $\operatorname{typ}(\Lambda)=\left(A_{2 p+1}, s, 2\right)$, then $\mathcal{C}=\left\langle\tau^{m_{\Delta}}\right\rangle\{(-1,1),(-2,1), \ldots,(-p, 1),(-1, n),(-2, n)$, $\ldots,(-p, n),(-p-1, p+1)\}$.

If $\Delta=D_{n}$ and $f$ is an integer, then $\{(-1,1),(-1, n-1),(-1, n)\} \subseteq \mathcal{C}$ (thus $\Lambda$ is "two-cornered" by the terminology in [4]).

If $\Delta=D_{3 m}$ and $f$ is not an integer, then $\{(0, n),(-(m-1), n),(-2(m-1), n)\} \subseteq \mathcal{C}$ (thus $\Lambda$ is "three-cornered" by the terminology in [4]).

If $\Delta=E_{6}$, then $\mathcal{C}=\left\langle\tau^{m_{\Delta}}\right\rangle\{(-1,1),(-2,1),(-1,5),(-2,5),(-1,6),(-6,1)\}$.

By this calculation we obtain the following.

Lemma 2.2. Assume that $\Delta=A_{n}(n \geq 1), D_{n}(n \geq 4)$ or $E_{6}$ (i.e. that $\eta$ is defined). Then $\mathcal{C}$ is stable under $\eta$ if and only if

$$
\operatorname{typ}(\Lambda) \notin\left\{\left(A_{n}, s / n, 1\right),\left(D_{3 m}, r / 3,1\right) \mid n, r, s \geq 1, m \geq 2,3 \nmid r\right\} .
$$

If $\Delta=D_{4}, \mathcal{C}$ is stable also under $\eta_{i}$ for all $i \in\{1,2,3,4,5\}$. 
Remark. This calculation of $\mathcal{C}$ makes up the omitted part in the proof of $[1$, Theorem 2.2(4), p. 194]. Namely, this shows that all the types listed in Proposition 1.1 really appear in examples.

\section{Stable Picard groups for Representative Algebras}

In this section we investigate stable self-equivalences of representative algebras listed in Appendix 2.

Definition 1. Let $\Lambda$ be an algebra. The set $\operatorname{StPic}(\Lambda)$ of natural isomorphism classes $[\phi]$ of stable equivalences $\phi: \underline{\bmod } \Lambda \rightarrow \underline{\bmod } \Lambda$ forms a group under the composition of functors, which is called the stable Picard group of $\Lambda$. The image of the canonical homomorphism $\operatorname{Pic}(\Lambda) \rightarrow \operatorname{StPic}(\Lambda)$ from the Picard group $\operatorname{Pic}(\Lambda)$ to $\operatorname{StPic}(\Lambda)$ is denoted by $\operatorname{Pic}^{\prime}(\Lambda)$. Thus for a $[\phi] \in \operatorname{StPic}(\Lambda),[\phi] \in \operatorname{Pic}^{\prime}(\Lambda)$ if and only if $\phi$ lifts to a Morita equivalence.

Remark. (1) As easily seen $\left[\Omega_{\Lambda}\right]$ commutes with all $[\phi] \in \operatorname{Pic}^{\prime}(\Lambda)$.

(2) If $\Lambda$ is a selfinjective algebra, then $-\otimes_{\Lambda} D \Lambda$ is a Morita equivalence and hence the Auslander-Reiten translation $\tau_{\Lambda}$ satisfies $\left[\tau_{\Lambda}\right]=\left[\Omega_{\Lambda}^{2} \circ\left(-\otimes_{\Lambda} D \Lambda\right)\right] \in \operatorname{Pic}^{\prime}(\Lambda)\left\langle\left[\Omega_{\Lambda}\right]\right\rangle$.

The purpose of this section is to prove the following.

Theorem 3.1. Let $\Lambda$ be a representative algebra of representation-finite standard selfinjective algebras listed in Appendix 2. If $\Lambda$ is not of type $\left(D_{3 m}, s / 3,1\right)$ with $m \geq 2$, $3 \nmid s$, then we have

$$
\operatorname{StPic}(\Lambda)=\operatorname{Pic}^{\prime}(\Lambda)\left\langle\left[\Omega_{\Lambda}\right]\right\rangle .
$$

If $\Lambda$ is of type $\left(D_{3 m}, s / 3,1\right)$ with $m \geq 2,3 \nmid s$, then

$$
\operatorname{StPic}(\Lambda)=\left(\operatorname{Pic}^{\prime}(\Lambda)\left\langle\left[\Omega_{\Lambda}\right]\right\rangle\right) \cup\left(\operatorname{Pic}^{\prime}(\Lambda)\left\langle\left[\Omega_{\Lambda}\right]\right\rangle\right)[H],
$$

where $H$ is a stable self-equivalence of $\Lambda$ induced from $\eta$, which satisfies $[H]^{2} \in \operatorname{Pic}^{\prime}(\Lambda)$.

For a representation-finite standard selfinjective algebra $\Lambda$, let ind $\Lambda$ be the full subcategory of $\bmod \Lambda$ formed by a fixed complete set of representatives of isoclasses of indecomposable $\Lambda$-modules. Identify ind $\Lambda$ with the mesh category $k\left(\Gamma_{\Lambda}\right)$ by a fixed isomorphism. Also identify the mesh category $k\left({ }_{s} \Gamma_{\Lambda}\right)$ with the stable category $k\left(\Gamma_{\Lambda}\right)$ by the equivalence $k\left({ }_{s} \Gamma_{\Lambda}\right) \rightarrow k\left(\Gamma_{\Lambda}\right)$ induced from the inclusion ${ }_{s} \Gamma_{\Lambda} \hookrightarrow \Gamma_{\Lambda}$. Then the stable category ind $\Lambda$ is identified with $k\left({ }_{s} \Gamma_{\Lambda}\right)$.

Lemma 3.2. Let $\Lambda$ and $\Pi$ be representation-finite standard selfinjective algebras, and

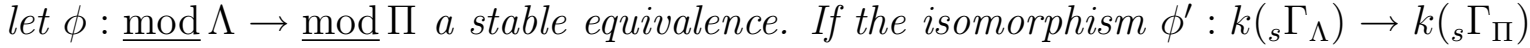
of the mesh categories induced from $\phi$ lifts to an isomorphism of the mesh categories $k\left(\Gamma_{\Lambda}\right) \rightarrow k\left(\Gamma_{\Pi}\right)$, then $\phi$ lifts to a Morita equivalence.

Proof. This is straitforward.

The following proposition makes it possible to prove Theorem 3.1 by a combinatorial argument. 
Proposition 3.3. Let $\Lambda$ and $\Pi$ be representation-finite standard selfinjective algebras,

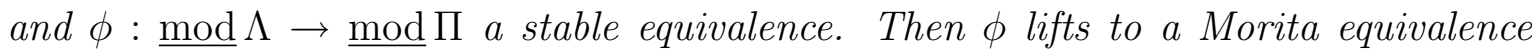
if and only if $\phi$ maps the configuration of $\Lambda$ into that of $\Pi$, i.e., for any projective indecomposable $\Lambda$-module $P$, there exists a projective indecomposable $\Pi$-module $Q$ such that $\phi(\operatorname{rad} P) \cong \operatorname{rad} Q$.

Proof. $(\Rightarrow)$. Assume that $\phi$ lifts to a Morita equivalence $\Phi$. Let $P$ be an indecomposable projective $\Lambda$-module. Then $Q:=\Phi(P)$ is an indecomposable projective $\Pi$-module. Since the inclusion map $\alpha: \operatorname{rad} P \rightarrow P$ is a source map, $\Phi(\alpha): \Phi(\operatorname{rad} P) \rightarrow Q$ is a source map. Hence $\Phi(\operatorname{rad} P) \cong \operatorname{rad} Q$ in $\bmod \Pi$. Thus $\phi(\operatorname{rad} P) \cong \operatorname{rad} Q$ in $\underline{\bmod } \Pi$.

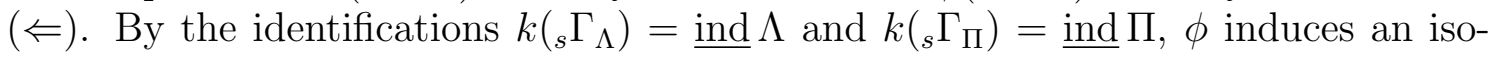
morphism $\phi^{\prime}: k\left({ }_{s} \Gamma_{\Lambda}\right) \rightarrow k\left({ }_{s} \Gamma_{\Pi}\right)$, and $\phi^{\prime}$ induces a quiver isomorphism $\psi:{ }_{s} \Gamma_{\Lambda} \rightarrow{ }_{s} \Gamma_{\Pi}$. Assume that $\phi$ maps the configuration of $\Lambda$ into that of $\Pi$. Let $P$ be an indecomposable projective $\Lambda$-module, and consider an almost split sequence

$$
0 \longrightarrow \operatorname{rad} P \stackrel{\left(\begin{array}{l}
f_{1} \\
f_{2}
\end{array}\right)}{\longrightarrow} P \oplus \operatorname{rad} P / \operatorname{soc} P \stackrel{\left(g_{1}, g_{2}\right)}{\longrightarrow} P / \operatorname{soc} P \longrightarrow 0 .
$$

By assumption $\phi(\operatorname{rad} P) \cong \operatorname{rad} Q$ for some indecomposable projective $\Pi$-module $Q:=$ $Q_{P}$. Then clearly $\phi(P / \operatorname{soc} P) \cong Q / \operatorname{soc} Q$. Since $g_{2} f_{2}=-g_{1} g_{1}: \operatorname{rad} P \rightarrow P / \operatorname{soc} P$ factors through the projective module $P$, any representative $h$ in $\phi\left(g_{2} f_{2}\right)$ factors through a projective $\Pi$-module. But since $Q$ is a projective cover of $Q / \operatorname{soc} Q, h$ factors through $Q$. Now denote the mesh corresponding to the almost split sequence above by

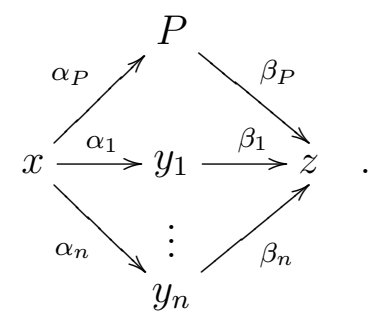

Then for each $i=1, \ldots, n$ we have

$$
\begin{aligned}
\phi^{\prime}\left(\alpha_{i}\right) & =s_{i} \overline{\psi\left(\alpha_{i}\right)}+\mu_{i} \\
\phi^{\prime}\left(\beta_{i}\right) & =t_{i} \overline{\psi\left(\beta_{i}\right)}+\nu_{i}
\end{aligned}
$$

for some $s_{i}, t_{i} \in k^{\times}:=k \backslash\{0\}$ and some $\mu_{i}, \nu_{i} \in \operatorname{rad}^{2} k\left(\Gamma_{\Pi}\right)$, where $\overline{(-)}$ denotes the coset modulo the mesh ideal. Further by the above $h:=\sum_{i=1}^{n} \phi^{\prime}\left(\beta_{i}\right) \phi^{\prime}\left(\alpha_{i}\right)$ in $\phi\left(\underline{g_{2} f_{2}}\right)$ factors through the projective cover $Q$ of $\psi(z)$, say $h=-\eta_{P} \zeta_{P}$. Then we have

$$
\eta_{P} \zeta_{P}+\sum_{i=1}^{n} \phi^{\prime}\left(\beta_{i}\right) \phi^{\prime}\left(\alpha_{i}\right)=0
$$

by which we can define a functor $\Phi: k\left(\Gamma_{\Lambda}\right) \rightarrow k\left(\Gamma_{\Pi}\right)$ by $\Phi:=\phi^{\prime}$ on ${ }_{s} \Gamma_{\Lambda}$; and $\Phi(P):=$ $Q_{P}$ and $\Phi\left(\alpha_{P}\right):=\zeta_{P}$ and $\Phi\left(\beta_{P}\right):=\eta_{P}$ for each projective vertex $P$ in $\Gamma_{\Lambda}$. Then by construction $\Phi$ is a lift of $\phi^{\prime}$. By Lemma 3.2 it is enough to show that $\Phi$ is an isomorphism. Since $\Phi$ induces a quiver isomorphism $\Psi: \Gamma_{\Lambda} \rightarrow \Gamma_{\Pi}$ that is an extension of $\psi$, we have $\operatorname{dim}_{k} k\left(\Gamma_{\Lambda}\right)(x, y)=\operatorname{dim}_{k} k\left(\Gamma_{\Pi}\right)(\Phi(x), \Phi(y))$ for all vertices $x, y$ of $\Gamma_{\Lambda}$. 
Thus we have only to show that $\Phi$ is full. For each projective vertex $P$ in $\Gamma_{\Lambda}$ we can write

$$
\begin{aligned}
& \zeta_{P}=s_{P} \overline{\Psi\left(\alpha_{P}\right)}+\mu_{P} \\
& \eta_{P}=t_{P} \overline{\Psi\left(\beta_{P}\right)}+\nu_{P}
\end{aligned}
$$

for some $s_{P}, t_{P} \in k$ and some $\mu_{P}, \nu_{P} \in \operatorname{rad}^{2} k\left(\Gamma_{\Pi}\right)$. Suppose that $s_{P} t_{P}=0$ for some $P$. Then (1), (2) and (3) show that

$$
\sum_{i=1}^{n} t_{i} s_{i} \overline{\psi\left(\beta_{i}\right) \psi\left(\alpha_{i}\right)} \in \operatorname{rad}^{3} k\left(\Gamma_{\Pi}\right) .
$$

But since each mesh relation is a sum of paths of length 2, this forces to have $\Psi\left(\beta_{P}\right) \Psi\left(\alpha_{P}\right)$ $=0$ in $k \Gamma_{\Pi}$, which gives a contradiction. Indeed the formula above yields

$$
\sum_{i=1}^{n} t_{i} s_{i} \psi\left(\beta_{i}\right) \psi\left(\alpha_{i}\right)-\xi=\sum_{j=1}^{m} r_{j} \delta_{j} m_{j} \gamma_{j}
$$

in $k \Gamma_{\Pi}(\psi x, \psi z)$ for some $r_{j} \in k^{\times}$, some paths $\gamma_{j}, \delta_{j}$ in $\Gamma_{\Pi}$ and some mesh relations $m_{j}$. We may assume that $\delta_{j} m_{j} \gamma_{j} \neq 0$ for all $j$. Let $m(\psi z):=\Psi\left(\beta_{P}\right) \Psi\left(\alpha_{P}\right)+\sum_{i=1}^{n} \psi \beta_{i} \psi \alpha_{i}$ be the mesh relation ending in $\psi z$. Comparing the terms of length 2 we get

$$
\sum_{i=1}^{n} t_{i} s_{i} \psi\left(\beta_{i}\right) \psi\left(\alpha_{i}\right)=r \cdot m(\psi z),
$$

where $r:=\sum_{j \in J} r_{j} \in k$ and $J:=\left\{j \in\{1, \ldots, m\} \mid\right.$ both $\delta_{j}$ and $\gamma_{j}$ are trivial paths $\}$, from which we get $\Psi\left(\beta_{P}\right) \Psi\left(\alpha_{P}\right)=0$ in the path category $k \Gamma_{\Pi}$, as desired. This contradiction implies that $s_{P} t_{P} \neq 0$, i.e., $s_{P} \neq 0$ and $t_{P} \neq 0$ for all projective vertices $P$ in $\Gamma_{\Lambda}$. This shows that $\overline{\Psi\left(\alpha_{P}\right)}, \overline{\Psi\left(\beta_{P}\right)} \in \Phi\left(\operatorname{rad} k\left(\Gamma_{\Lambda}\right)\right)+\operatorname{rad}^{2} k\left(\Gamma_{\Pi}\right)$. Therefore since $\Phi=\phi^{\prime}$ on ${ }_{s} \Gamma_{\Lambda}$, we have $\operatorname{rad} k\left(\Gamma_{\Pi}\right) \leq \Phi\left(\operatorname{rad} k\left(\Gamma_{\Lambda}\right)\right)+\operatorname{rad}^{2} k\left(\Gamma_{\Pi}\right) . \operatorname{Thus} \operatorname{rad} k\left(\Gamma_{\Pi}\right) \leq$ $\Phi\left(\operatorname{rad} k\left(\Gamma_{\Lambda}\right)\right)$ because $\operatorname{rad} k\left(\Gamma_{\Pi}\right)$ is nilpotent. Hence $\Phi$ is full.

Remark. Let $\phi$ be a stable equivalence between selfinjective algebras. If $\phi$ is of Morita type, then (1) by Linkelmann [7, section 2] $\phi$ lifts to a Morita equivalence if and only if $\phi$ maps simples to simples, and (2) as easily seen the latter is equivalent to saying that $\phi$ maps the configuration into configuration. Therefore when $\phi$ is of Morita type, Proposition 3.3 necessarily holds. This suggests us that all stable equivalences between representation-finite standard selfinjective algebras would be of Morita type.

Proof of Theorem 3.1. Let $\Lambda$ be a representative algebra of representation-finite standard selfinjective algebras listed in Appendix 2. Let $\phi: \underline{\bmod } \Lambda \rightarrow \underline{\bmod } \Lambda$ be a stable equivalence. As above $\phi$ induces an automorphism $\phi^{\prime}$ of $k\left({ }_{s} \Gamma_{\Lambda}\right)$. Put $\operatorname{typ}(\Lambda)=$ $(\Delta, f, t)$. Then ${ }_{s} \Gamma_{\Lambda}$ is identified with $\mathbb{Z} \Delta /\langle g\rangle$ for some admissible automorphism $g$ of $\mathbb{Z} \Delta$ as in section 1 ; and $\phi^{\prime}$ induces a quiver automorphism $\psi$ of $\mathbb{Z} \Delta /\langle g\rangle$. Lift $\psi$ to an 
automorphism $\tilde{\psi}$ of $\mathbb{Z} \Delta$, thus we have a commutative diagram

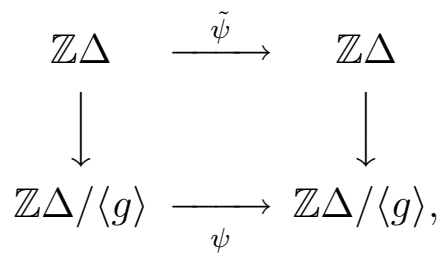

where the vertical morphisms are the canonical one. We keep the notation in section 2. By Proposition 2.1 we have $\tilde{\psi}=\tau^{a} \rho$ for some $\rho \in\left\{\mathbb{1}, \eta, \eta_{1}, \eta_{2}, \eta_{3}, \eta_{4}, \eta_{5}\right\}$ and some $a \in \mathbb{Z}$.

Suppose that $\operatorname{typ}(\Lambda) \notin\left\{\left(A_{n}, s / n, 1\right),\left(D_{3 m}, r / 3,1\right) \mid n, r, s \geq 1, m \geq 2,3 \nmid r\right\}$ or $\rho=\mathbb{1}$. Then by Lemma $2.2, \mathcal{C}$ is stable under $\rho$, i.e., under $\tau^{-a} \tilde{\psi}$, which means that $\xi:=\tau_{\Lambda}^{-a} \phi$ maps the configuration of $\Lambda$ into itself. Therefore by Proposition 3.3, $\xi$ lifts to a Morita equivalence, i.e., $[\xi] \in \operatorname{Pic}^{\prime}(\Lambda)$. Hence $[\phi]=\left[\tau_{\Lambda}^{a} \xi\right] \in \operatorname{Pic}^{\prime}(\Lambda)\left\langle\left[\Omega_{\Lambda}\right]\right\rangle$.

Next suppose that $\rho \neq \mathbb{1}$ and $\operatorname{typ}(\Lambda)=\left(A_{n}, s / n, 1\right)$ for some $n, s \geq 1$. Then $\rho=\eta$, $\Lambda$ is the selfinjective Nakayama algebra of Loewy length $n+1$ with exactly $s$ isoclasses of simple modules, and $\mathcal{C}=\{(i, n) \mid i \in \mathbb{Z}\}$. In this case both $\phi$ and the loop functor $\Omega_{\Lambda}$ maps indecomposable modules of length $x$ to indecomposable modules of length $n+1-x$ for all $1 \leq x \leq n$. Therefore $\xi:=\Omega_{\Lambda} \phi$ maps the configuration $\{\langle g\rangle(i, n) \mid i \in \mathbb{Z}\}$ of $\Lambda$ into itself. Hence $[\xi] \in \operatorname{Pic}^{\prime}(\Lambda)$ by Proposition 3.3 and $[\phi]=\left[\Omega_{\Lambda}^{-1} \xi\right] \in \operatorname{Pic}^{\prime}(\Lambda)\left\langle\left[\Omega_{\Lambda}\right]\right\rangle$.

Finally suppose that $\rho \neq \mathbb{1}$ and $\operatorname{typ}(\Lambda)=\left(D_{3 m}, s / 3,1\right)$ for some $m \geq 2$ and $s$ with $3 \nmid s$. Then $\rho=\eta$ and $[\phi] \in\left(\operatorname{Pic}^{\prime}(\Lambda)\left\langle\left[\Omega_{\Lambda}\right]\right\rangle\right)[H]$ by the same argument as above. It follows from $\eta^{2}=\mathbb{1}$ that $[H]^{2} \in \operatorname{Pic}^{\prime}(\Lambda)$.

Remark. (1) The product $\operatorname{Pic}^{\prime}(\Lambda)\left\langle\left[\Omega_{\Lambda}\right]\right\rangle$ is not necessarily a direct product. For instance, if $\operatorname{typ}(\Lambda)=\left(A_{n}, 1,1\right)$ with $n \geq 2$, then $\Lambda$ is symmetric, and $[\mathbb{1}] \neq$ $\left[\tau_{\Lambda}\right]=\left[\Omega_{\Lambda}^{2}\right]$ maps the configuration of $\Lambda$ into itself and is in $\operatorname{Pic}^{\prime}(\Lambda) \cap\left\langle\left[\Omega_{\Lambda}\right]\right\rangle$ by Proposition 3.3.

(2) The reason why Main Theorem is open for the types $\left(D_{3 m}, s / 3,1\right)$ with $m \geq 2$, $3 \nmid s \geq 1$ is that in this case we could not make it clear whether the stable equivalence $H$ induced by $\eta$ lifts to a standard derived equivalence or not.

\section{Proof of Main Theorem}

The following is easily verified.

Lemma 4.1. Let $\Lambda, \Lambda^{\prime}$ and $\Lambda^{\prime \prime}$ be selfinjective algebras.

(1) If $\phi: \underline{\bmod } \Lambda \rightarrow \underline{\bmod } \Lambda^{\prime}$ is a stable equivalence that lifts to a standard derived equivalence, then so does any quasi-inverse of $\phi$; and

(2) If both $\phi: \underline{\bmod } \Lambda \rightarrow \underline{\bmod } \Lambda^{\prime}$ and $\phi^{\prime}: \underline{\bmod } \Lambda^{\prime} \rightarrow \underline{\bmod } \Lambda^{\prime \prime}$ are stable equivalences that lift to standard derived equivalences, then so does the composite $\phi^{\prime} \phi: \underline{\bmod } \Lambda \rightarrow \underline{\bmod } \Lambda^{\prime \prime}$.

Lemma 4.2. Let $\Lambda$ and $\Pi$ be selfinjective algebras. Then

(1) The loop functor $\Omega_{\Lambda}$ lifts to a standard derived equivalence;

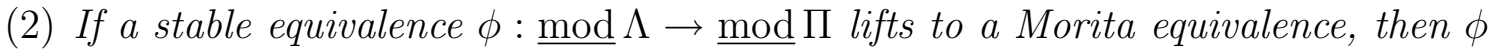
lifts to a standard derived equivalence; and 
Proof. (1) As easily verified $\Omega_{\Lambda}$ lifts to the standard derived equivalence $-\stackrel{\mathbf{L}}{\otimes_{\Lambda}}(\Lambda[1])$.

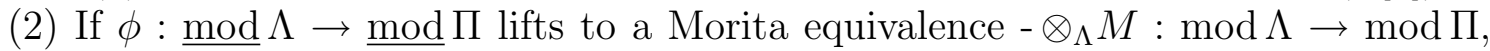
then $M$ as a complex concentrated in degree zero is a twosided tilting complex and

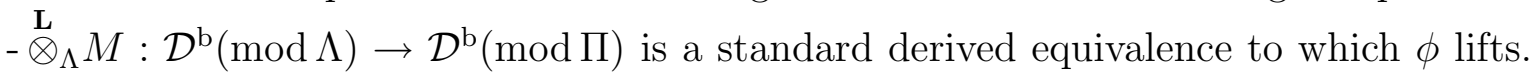

By Theorem 3.1 and Lemma 4.2 we obtain the following.

Theorem 4.3. Let $\Lambda$ be a representative algebra of representation-finite selfinjective algebras listed in Appendix 2. If $\Lambda$ is not of type $\left(D_{3 m}, s / 3,1\right)$ with $m \geq 2,3 \nmid s$, then every stable self-equivalence of $\Lambda$ lifts to a standard derived equivalence.

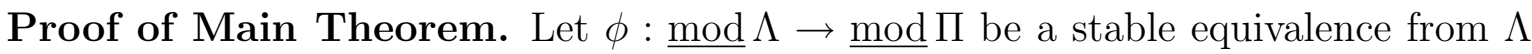
to $\Pi$. Then $\operatorname{can}(\Lambda)=\operatorname{can}(\Pi)=$ : $\Gamma$. By Theorem $1.2 \Lambda$ and $\Gamma$ (resp. $\Pi$ and $\Gamma$ ) are derived equivalent. Therefore there exists a standard derived equivalence $\mathcal{D}^{\mathrm{b}}(\bmod \Lambda) \rightarrow$ $\mathcal{D}^{\mathrm{b}}(\bmod \Gamma)\left(\operatorname{resp} . \quad \mathcal{D}^{\mathrm{b}}(\bmod \Pi) \rightarrow \mathcal{D}^{\mathrm{b}}(\bmod \Gamma)\right)$ that induces a stable equivalence $\zeta$ : $\underline{\bmod } \Lambda \rightarrow \underline{\bmod } \Gamma($ resp. $\eta: \underline{\bmod } \Pi \rightarrow \underline{\bmod } \Gamma)$. Thus both $\zeta$ and $\eta$ lift to standard derived equivalences. Then by Theorem 4.3, $\xi:=\eta \phi \zeta^{-1}: \underline{\bmod } \Gamma \rightarrow \underline{\bmod } \Gamma$ lifts to a standard derived equivalence because $\Gamma$ is a representative algebra. Hence $\phi=\eta^{-1} \xi \zeta$ lifts to a standard derived equivalence by Lemma 4.1.

\section{ApPendix 1: Stable Equivalences And Standard DERIVED EQUivalences}

In this section we show that if a stable equivalence $\phi$ of selfinjective algebras lifts to a standard derived equivalence $\Phi$, then the stable equivalence $\phi^{\prime}$ of Morita type obtained from $\Phi$ as given in Rickard [10, Corollary 5.5] coincides with $\phi$. But giving a proof of this is almost equivalent to giving a proof of the fact that $\phi^{\prime}$ is of Morita type. Therefore we here give a simple proposition that produces both proofs.

First recall the definition of $\eta_{\Lambda}: \mathcal{D}^{\mathrm{b}}(\bmod \Lambda) \rightarrow \underline{\bmod } \Lambda$ for a selfinjective algebra $\Lambda$. Denote by $\mathcal{H}_{\mathrm{b}}^{-}(\operatorname{pro} \Lambda)$ the full subcategory of the right bounded homotopy category $\mathcal{H}^{-}$(pro $\left.\Lambda\right)$ of pro $\Lambda$ consisting of objects with bounded homology. As well-known there exists an equivalence $\boldsymbol{p}_{\Lambda}: \mathcal{D}^{\mathrm{b}}(\bmod \Lambda) \rightarrow \mathcal{H}_{\mathrm{b}}^{-}(\operatorname{pro} \Lambda)$ of triangulated categories such that there is a quasi-isomorphism $\boldsymbol{p}_{\Lambda}\left(A^{\bullet}\right) \rightarrow A^{\bullet}$ for all $A^{\cdot} \in \mathcal{D}^{\mathrm{b}}(\bmod \Lambda)$. Now let $A^{\bullet} \in \mathcal{D}^{\mathrm{b}}(\bmod \Lambda)$ and put $\boldsymbol{p}_{\Lambda}\left(A^{\bullet}\right)=P^{\cdot}=\left(P^{i}, d_{P}^{i}\right)_{i \in \mathbb{Z}}$. Then we can choose an integer $a<0$ such that $H^{i}\left(P^{\bullet}\right)=0$ for all $i \leq a$, and define $\eta_{\Lambda}\left(A^{\bullet}\right):=\Omega_{\Lambda}^{a}\left(\operatorname{Im} d_{P}^{a}\right)$. This is known to be well-defined ([9]). Thus $\eta_{\Lambda}\left(A^{*}\right)=M$ if and only if we have an exact sequence

$$
0 \rightarrow \operatorname{Im} d_{P}^{a} \rightarrow Q^{a+1} \rightarrow \cdots \rightarrow Q^{0} \rightarrow M \rightarrow 0
$$

in $\bmod \Lambda$ with each $Q^{i}$ in $\operatorname{pro} \Lambda$.

Before stating the proposition, we recall general facts in the following three lemmas. The following is well-known.

Lemma 5.1. Let $K$ be a commutative ring and $R$ and $S$ be $K$-algebras. If $M$ is an $R$ $S$-bimodule, $X$ is a right $R$-module and $Y$ is a right $S$-module, then we have a natural isomorphism

$$
\operatorname{Hom}_{S}\left(X \otimes_{R} M, Y\right) \cong \operatorname{Hom}_{R^{\mathrm{op}} \otimes_{K} S}\left(M, \operatorname{Hom}_{K}(X, Y)\right) .
$$


In particular, if $K$ is a field and $M$ is a projective $R$-S-bimodule, then $X \otimes_{R} M$ is a projective right $S$-module for all right $R$-modules $X$.

As for the converse of the last statement we recall the following from Auslander and Reiten [3, Corollary 3.3].

Lemma 5.2. Let $K$ be a perfect field and $R$ and $S$ be finite-dimensional $K$-algebras. Then for a finite-dimensional $R$-S-bimodule $M$ the following are equivalent.

(1) $M$ is a projective bimodule.

(2) $M$ is a projective right $S$-module and $X \otimes_{R} M$ are projective right $S$-modules for all finite-dimensional right $R$-modules $X$.

In particular, if $M$ is projective as an $R$-S-bimodule, then so is $X \otimes_{R} M$ for any finite-dimensional right $R$-module $X$.

Lemma 5.3. Let $K$ be a field and $R$ and $S$ be finite-dimensional $K$-algebras. If $X$ is a finite-dimensional injective left $R$-module and $Y$ is a finite-dimensional injective right $S$-module, then $X \otimes_{K} Y$ is an injective $R$-S-bimodule. In particular, if both $R$ and $S$ are selfinjective algebras, then so is $R^{\mathrm{op}} \otimes_{K} S$.

Proof. The assertion follows from the following isomorphisms:

$$
\begin{aligned}
\operatorname{Hom}_{R^{\mathrm{op}} \otimes_{K} S}\left(-, X \otimes_{K} Y\right) & \cong \operatorname{Hom}_{R^{\mathrm{op}} \otimes_{K} S}\left(-, \operatorname{Hom}_{K}\left(\operatorname{Hom}_{K}(X, K), Y\right)\right) \\
& \cong \operatorname{Hom}_{S}\left(\operatorname{Hom}_{K}(X, K) \otimes_{R^{-}}, Y\right) .
\end{aligned}
$$

The last isomorphism is given by Lemma 5.1.

We now prove the following.

Proposition 5.4. Let $\Gamma, \Lambda$, $\Pi$ be selfinjective algebras and $X^{\cdot} \in \mathcal{D}^{\mathrm{b}}\left(\bmod \Lambda^{\mathrm{op}} \otimes_{k} \Pi\right)$ be a twosided tilting complex. Then $M:=\eta_{\Lambda^{\mathrm{op}} \otimes_{k} \Pi}\left(X^{*}\right) \in \underline{\bmod } \Lambda^{\mathrm{op}} \otimes_{k} \Pi$ is projective both in $\bmod \Lambda^{\mathrm{op}}$ and in $\bmod \Pi$ and the diagram

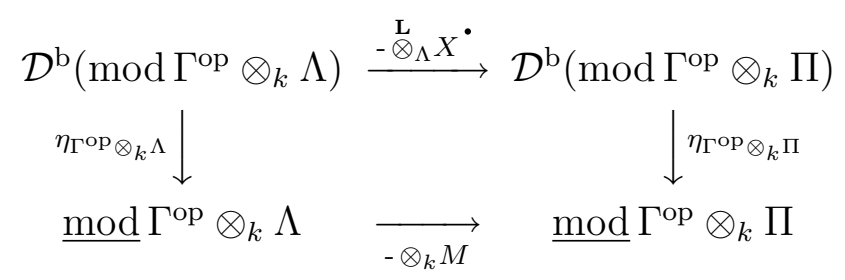

commutes up to natural isomorphisms.

Proof. First note that algebras $\Lambda^{\mathrm{op}} \otimes_{k} \Pi, \Gamma^{\mathrm{op}} \otimes_{k} \Lambda, \Gamma^{\mathrm{op}} \otimes_{k} \Pi$ are selfinjective algebras by Lemma 5.3. Put $\Delta^{\cdot}=\left(\Delta^{i}, d_{\Delta}^{i}\right)_{i \in \mathbb{Z}}:=\boldsymbol{p}_{\Lambda^{\mathrm{op}} \otimes_{k} \Pi}\left(X^{\bullet}\right)$ and choose an integer $a<0$ such that $H^{i}\left(\Delta^{*}\right)=0$ for all $i \leq a$. It is enough to show that

$$
A \otimes_{k} M \cong \eta_{\Gamma \mathrm{op} \otimes_{k} \Pi}\left(A \stackrel{\mathbf{L}}{\otimes_{\Lambda}} X^{\bullet}\right)
$$

for all $A \in \underline{\bmod } \Gamma^{\cdot} \otimes_{k} \Lambda$ natural in $A$. Let $A \in \underline{\bmod } \Gamma^{\cdot} \otimes_{k} \Lambda$. Then

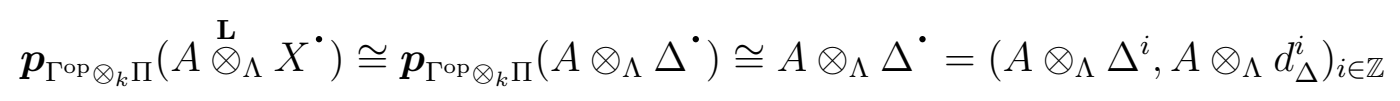


because $A \otimes_{\Lambda} \Delta^{i} \in$ pro $\Gamma^{\mathrm{op}} \otimes_{k} \Pi$ for all $i \in \mathbb{Z}$ by Lemma 5.2. By definition of $M$ we have an exact sequence

$$
0 \rightarrow \operatorname{Im} d_{\Delta}^{a} \rightarrow \Theta^{a+1} \rightarrow \cdots \rightarrow \Theta^{0} \rightarrow M \rightarrow 0
$$

in $\bmod \Lambda^{\mathrm{op}} \otimes_{k} \Pi$ with $\Theta^{i} \in \operatorname{pro} \Lambda^{\mathrm{op}} \otimes_{k} \Pi$ for all $i \in\{0, \ldots, a+1\}$. Since all $\Delta^{i}, \Theta^{i}$ are both in pro $\Lambda^{\text {op }}$ and in pro $\Pi$, we have ${ }_{\Lambda} M \cong \eta_{\Lambda^{\text {op }}}\left(X^{*}\right)$ and $M_{\Pi} \cong \eta_{\Pi}\left(X^{*}\right)$. But since ${ }_{\Lambda} X^{\bullet} \in \mathcal{H}^{\mathrm{b}}\left(\operatorname{pro} \Lambda^{\mathrm{op}}\right)$ and $X_{\Pi}^{\cdot} \in \mathcal{H}^{\mathrm{b}}(\operatorname{pro} \Pi)$, we have $\eta_{\Lambda_{\mathrm{op}}}\left(X^{\bullet}\right)=0 \operatorname{in} \underline{\bmod } \Lambda^{\text {op }}$ and $\eta_{\Pi}\left(X^{\bullet}\right)=0$ in $\underline{\bmod } \Pi$. Thus ${ }_{\Lambda} M \in \operatorname{pro} \Lambda^{\text {op }}$ and $M_{\Pi} \in$ pro $\Pi$. Hence the sequence $(*)$ is a composite of short exact sequences that split in $\bmod \Lambda^{\mathrm{op}}$, and $\operatorname{Im} d_{\Delta}^{a}$ is in $\operatorname{pro} \Lambda^{\mathrm{op}}$. Therefore $H^{i}\left(A \otimes_{\Lambda} \Delta^{*}\right)=0$ for all $i \leq a$ and we have an exact sequence

$$
0 \rightarrow \operatorname{Im}\left(A \otimes_{\Lambda} d_{\Delta}^{a}\right)=A \otimes_{\Lambda} \operatorname{Im} d_{\Delta}^{a} \rightarrow A \otimes_{\Lambda} \Theta^{a+1} \rightarrow \cdots \rightarrow A \otimes_{\Lambda} \Theta^{0} \rightarrow A \otimes_{\Lambda} M \rightarrow 0
$$

in $\bmod \Gamma^{\mathrm{op}} \otimes_{k} \Pi$ with each $A \otimes_{\Lambda} \Theta^{i} \in \operatorname{pro} \Gamma^{\mathrm{op}} \otimes_{k} \Pi$ by Lemma 5.2. Hence we have $A \otimes_{\Lambda} M \cong \eta_{\Gamma^{\mathrm{op}} \otimes_{k} \Pi}\left(A \stackrel{\mathbf{L}}{\otimes_{\Lambda}} X^{*}\right)$, which is natural in $A$ by construction.

Proposition 5.4 gives a simple proof of the construction of a stable equivalence of Morita type from a standard derived equivalence that is due to Rickard [10, Corollary $5.5]$.

Corollary 5.5. Let $\Lambda$ and $\Pi$ be selfinjective algebras. If ${ }_{\Lambda} X_{\Pi}^{*}$ and ${ }_{\Pi} Y_{\Lambda}^{*}$ are twosided tilting complexes giving a standard derived equivalence between $\Lambda$ and $\Pi$, then $M:=$ $\eta_{\Lambda^{\mathrm{op}} \otimes_{k} \Pi}\left(X^{*}\right)$ and $N:=\eta_{\Pi^{\mathrm{op}} \otimes_{k} \Lambda}\left(Y^{*}\right)$ give a stable equivalence of Morita type between $\Lambda$ and $\Pi$.

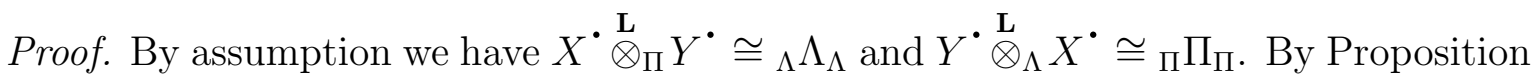
$5.4_{\Lambda} M, M_{\Pi},{ }_{\Pi} N$ and $N_{\Lambda}$ are projective and we have a diagram

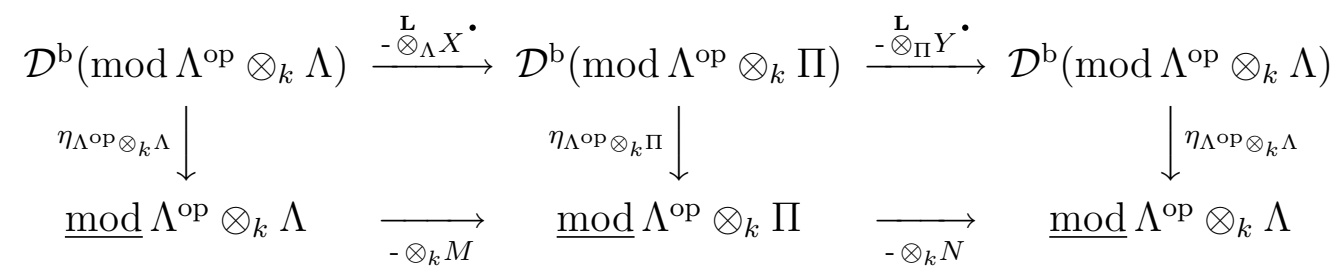

that commutes up to natural isomorphisms. This implies $M \otimes_{\Pi} N \cong \Lambda \otimes_{\Lambda} M \otimes_{\Pi}$ $N \cong{ }_{\Lambda} \Lambda_{\Lambda}$ in $\underline{\bmod } \Lambda^{\text {op }} \otimes_{k} \Lambda$. Hence $M \otimes_{\Pi} N \cong{ }_{\Lambda} \Lambda_{\Lambda} \oplus P$ in $\bmod \Lambda^{\text {op }} \otimes_{k} \Lambda$ for some $P \in \operatorname{pro} \Lambda^{\text {op }} \otimes_{k} \Lambda$. Similarly $N \otimes_{\Lambda} N \cong{ }_{\Pi} \Pi_{\Pi} \oplus Q$ in $\bmod \Pi^{\text {op }} \otimes_{k} \Pi$ for some $Q \in$ pro $\Pi^{\mathrm{op}} \otimes_{k} \Pi$.

Proposition 5.4 implies the statement in the beginning of this section.

Corollary 5.6. Let $\Lambda$ and $\Pi$ be selfinjective algebras and $\phi$ a stable equivalence from $\Lambda$ to $\Pi$. If $\phi$ lifts to a standard derived equivalence $\Phi$ from $\Lambda$ to $\Pi$, then $\phi$ is naturally isomorphic to the stable equivalence obtained by $\Phi$, and therefore $\phi$ is of Morita type. In particular, both $\Omega_{\Lambda}$ and $\tau_{\Lambda}$ are of Morita type. 
Proof. Put $\Phi=-\stackrel{\mathrm{L}}{\otimes_{\Lambda}} X^{\bullet}$ for some twosided tilting complex $X^{\bullet} \in \mathcal{D}^{\mathrm{b}}\left(\bmod \Lambda^{\mathrm{op}} \otimes_{k} \Pi\right)$ and put $M=\eta_{\Lambda^{\mathrm{op}} \otimes_{k} \Pi}\left(X^{\bullet}\right)$. Then by applying Proposition 5.4 for $\Gamma^{\mathrm{op}}=k$ we have a diagram

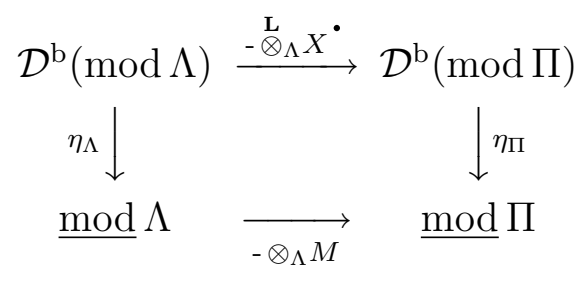

that commutes up to natural isomorphisms. Thus $-\otimes_{\Lambda} M$ is induced from $-\stackrel{\mathbf{L}}{\otimes_{\Lambda}} X^{\cdot}$. Therefore $\phi$ and $-\otimes_{k} M$ are naturally isomorphic.

Remark. For a selfinjective algebra $\Lambda$, it is not hard to show directly that the $\Lambda$ - $\Lambda$ bimodules $\Omega_{\Lambda^{\mathrm{op}} \otimes_{k} \Lambda}(\Lambda)$ and $\Omega_{\Lambda^{\mathrm{op}} \otimes_{k} \Lambda}^{-1}(\Lambda)$ satisfy the properties to show that $\Omega_{\Lambda}$ is of Morita type (see [2, Lemma 4.4]). 


\section{Appendix 2: Quiver PRESEntations of REPRESEntatives}

For the benefit of the reader we present the representative algebras of representationfinite selfinjective algebras by quivers with relations in this section.

6.1. Standard representatives. For each type $(\Delta, f, t)$ the algebra $\Lambda(\Delta, f, t)$ is given as follows.

6.1.1. $\left(A_{n}, s / n, 1\right)$ with $s, n \geq 1 . \Lambda\left(A_{n}, s / n, 1\right)$ is the selfinjective Nakayama algebra $N_{s, n}$ with exactly $s$ isoclasses of simples and of Loewy length $n+1$; namely this is given by the quiver $Q\left(N_{s, n}\right)$ with relations $R\left(N_{s, n}\right)$ below.

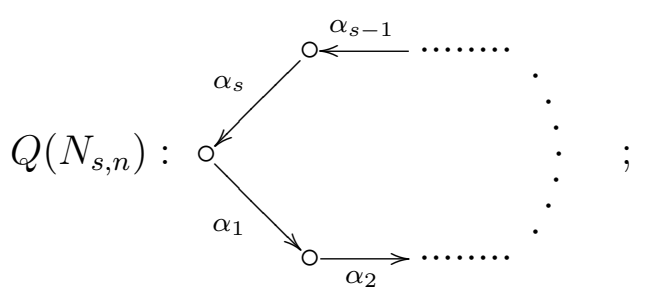

$R\left(N_{s, n}\right): \alpha_{i+n} \cdots \alpha_{i+1} \alpha_{i}=0$ for all $i \in\{1,2, \ldots, s\}=\mathbb{Z} /\langle s\rangle$.

6.1.2. $\left(A_{2 p+1}, s, 2\right)$ with $s, p \geq 1$. $\Lambda\left(A_{2 p+1}, s, 2\right)$ is the "canonical Möbius" algebra $M_{p, s}$ defined by the quiver $Q\left(M_{p, s}\right)$ with relations $R\left(M_{p, s}\right)$ below.

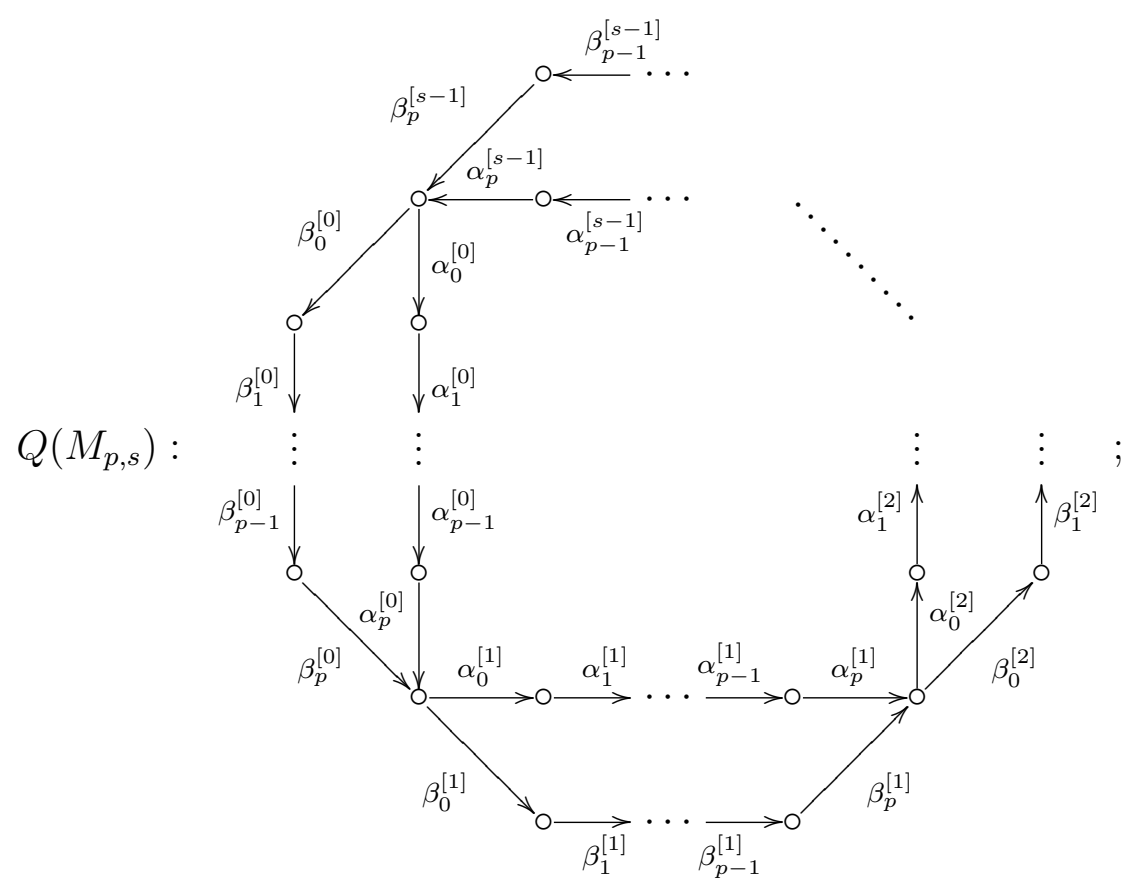

$R\left(M_{p, s}\right)$ :

(i) $\alpha_{p}^{[i]} \cdots \alpha_{0}^{[i]}=\beta_{p}^{[i]} \cdots \beta_{0}^{[i]}$ for all $i \in\{0, \ldots, s-1\}$;

(ii) $\beta_{0}^{[i+1]} \alpha_{p}^{[i]}=0, \alpha_{0}^{[i+1]} \beta_{p}^{[i]}=0$ for all $i \in\{0, \ldots, s-2\}$, and $\alpha_{0}^{[0]} \alpha_{p}^{[s-1]}=0, \beta_{0}^{[0]} \beta_{p}^{[s-1]}=$ 0 ; and

(iii) Paths of length $p+2$ are equal to 0 . 
6.1.3. $\left(D_{n}, s, 1\right)$ with $n \geq 4, s \geq 1 . \Lambda\left(D_{n}, s, 1\right)$ is given by the quiver $Q\left(D_{n}, s\right)$ with relations $R\left(D_{n}, s, 1\right)$ below.

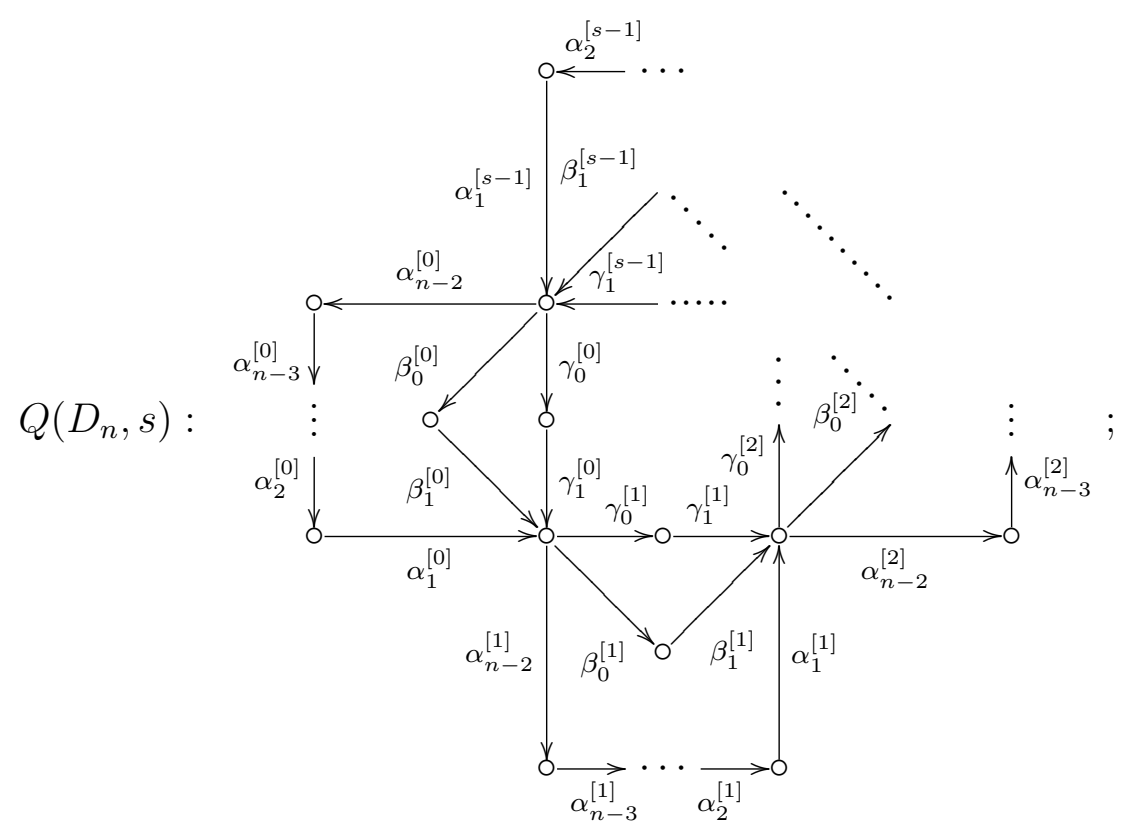

$R\left(D_{n}, s, 1\right)$ :

(i) $\alpha_{1}^{[i]} \alpha_{2}^{[i]} \cdots \alpha_{n-2}^{[i]}=\beta_{1}^{[i]} \beta_{0}^{[i]}=\gamma_{1}^{[i]} \gamma_{0}^{[i]}$ for all $i \in\{0, \ldots, s-1\}$;

(ii) For all $i \in\{0, \ldots, s-1\}=\mathbb{Z} /\langle s\rangle$,

$$
\left\{\begin{aligned}
\beta_{0}^{[i+1]} \alpha_{1}^{[i]}=0, & \gamma_{0}^{[i+1]} \alpha_{1}^{[i]}=0, \\
\alpha_{n-2}^{[i+1]} \beta_{1}^{[i]}=0, & \alpha_{n-2}^{[i+1]} \gamma_{1}^{[i]}=0, \\
\gamma_{0}^{[i+1]} \beta_{1}^{[i]}=0, & \beta_{0}^{[i+1]} \gamma_{1}^{[i]}=0 ; \text { and }
\end{aligned}\right.
$$

(iii) For all $i \in\{0, \ldots, s-1\}=\mathbb{Z} /\langle s\rangle$ and for all $j \in\{1, \ldots, n-2\}=\mathbb{Z} /\langle n-2\rangle$,

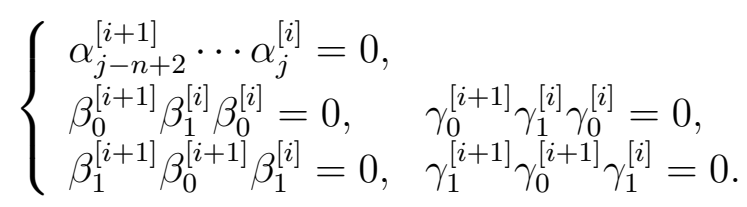

(i.e. " $\alpha$-paths" of length $n-1$ are equal to 0 , " $\beta$-paths" of length 3 are equal to 0 and " $\gamma$-paths" of length 3 are equal to 0 ).

6.1.4. $\left(D_{n}, s, 2\right)$ with $n \geq 4, s \geq 1$. $\Lambda\left(D_{n}, s, 2\right)$ is given by the quiver $Q\left(D_{n}, s\right)$ above with relations $R\left(D_{n}, s, 2\right)$ below.

$R\left(D_{n}, s, 2\right)$ :

(i) The same as $R\left(D_{n}, s, 1\right)(\mathrm{i})$;

(ii) For all $i \in\{0, \ldots, s-1\}=\mathbb{Z} /\langle s\rangle$,

$$
\left\{\begin{array}{cc}
\beta_{0}^{[i+1]} \alpha_{1}^{[i]}=0, & \gamma_{0}^{[i+1]} \alpha_{1}^{[i]}=0, \\
\alpha_{n-2}^{[i+1]} \beta_{1}^{[i]}=0, & \alpha_{n-2}^{[i+1]} \gamma_{1}^{[i]}=0,
\end{array}\right.
$$


and for all $i \in\{0, \ldots, s-2\}$,

$$
\begin{array}{ll}
\gamma_{0}^{[i+1]} \beta_{1}^{[i]}=0, & \beta_{0}^{[i+1]} \gamma_{1}^{[i]}=0 \\
\beta_{0}^{[0]} \beta_{1}^{[s-1]}=0, & \gamma_{0}^{[0]} \gamma_{1}^{[s-1]}=0 ; \text { and }
\end{array}
$$

(iii) $\alpha$-paths of length $n-1$ are equal to 0 , and for all $i \in\{0, \ldots, s-2\}$,

$$
\begin{gathered}
\begin{cases}\beta_{0}^{[i+1]} \beta_{1}^{[i]} \beta_{0}^{[i]}=0, & \gamma_{0}^{[i+1]} \gamma_{1}^{[i]} \gamma_{0}^{[i]}=0, \\
\beta_{1}^{[i+1]} \beta_{0}^{[i+1]} \beta_{1}^{[i]}=0, & \gamma_{1}^{[i+1]} \gamma_{0}^{[i+1]} \gamma_{1}^{[i]}=0, \text { and }\end{cases} \\
\begin{cases}\gamma_{0}^{[0]} \beta_{1}^{[s-1]} \beta_{0}^{[s-1]}=0, & \beta_{0}^{[0]} \gamma_{1}^{[s-1]} \gamma_{0}^{[s-1]}=0, \\
\gamma_{1}^{[0]} \gamma_{0}^{[0]} \beta_{1}^{[s-1]}=0, & \beta_{1}^{[0]} \beta_{0}^{[0]} \gamma_{1}^{[s-1]}=0\end{cases}
\end{gathered}
$$

6.1.5. $\left(D_{4}, s, 3\right)$ with $s \geq 1 . \Lambda\left(D_{4}, s, 3\right)$ is given by the quiver $Q\left(D_{4}, s\right)$ above with relations $R\left(D_{4}, s, 3\right)$ below.

$R\left(D_{4}, s, 3\right):\left(\right.$ put $\alpha_{0}^{[i]}:=\alpha_{2}^{[i]}$ for all $\left.i\right)$

(i) The same as $R\left(D_{4}, s, 1\right)(\mathrm{i})$;

(ii) For all $i \in\{0, \ldots, s-2\}$,

$$
\begin{gathered}
\begin{cases}\beta_{0}^{[i+1]} \alpha_{1}^{[i]}=0, & \gamma_{0}^{[i+1]} \alpha_{1}^{[i]}=0, \\
\alpha_{0}^{[i+1]} \beta_{1}^{[i]}=0, & \gamma_{0}^{[i+1]} \beta_{1}^{[i]}=0, \\
\alpha_{0}^{[i+1]} \gamma_{1}^{[i]}=0, & \beta_{0}^{[i+1]} \gamma_{1}^{[i]}=0, \text { and }\end{cases} \\
\begin{cases}\alpha_{0}^{[0]} \alpha_{1}^{[s-1]}=0, & \gamma_{0}^{[0]} \alpha_{1}^{[s-1]}=0, \\
\alpha_{0}^{[0]} \beta_{1}^{[s-1]}=0, & \beta_{0}^{[0]} \beta_{1}^{[s-1]}=0, \\
\beta_{0}^{[0]} \gamma_{1}^{[s-1]}=0, & \gamma_{0}^{[0]} \gamma_{1}^{[s-1]}=0 ; \text { and }\end{cases}
\end{gathered}
$$

(iii) Paths of length 3 are 0 . 
6.1.6. $\left(D_{3 m}, s / 3,1\right)$ with $m \geq 2$ and $3 \nmid s \geq 1 . \Lambda\left(D_{3 m}, s / 3,1\right)$ is given by the quiver $Q\left(D_{3 m}, s / 3\right)$ with relations $R\left(D_{3 m}, s / 3\right)$ below. For $s \geq 2$,

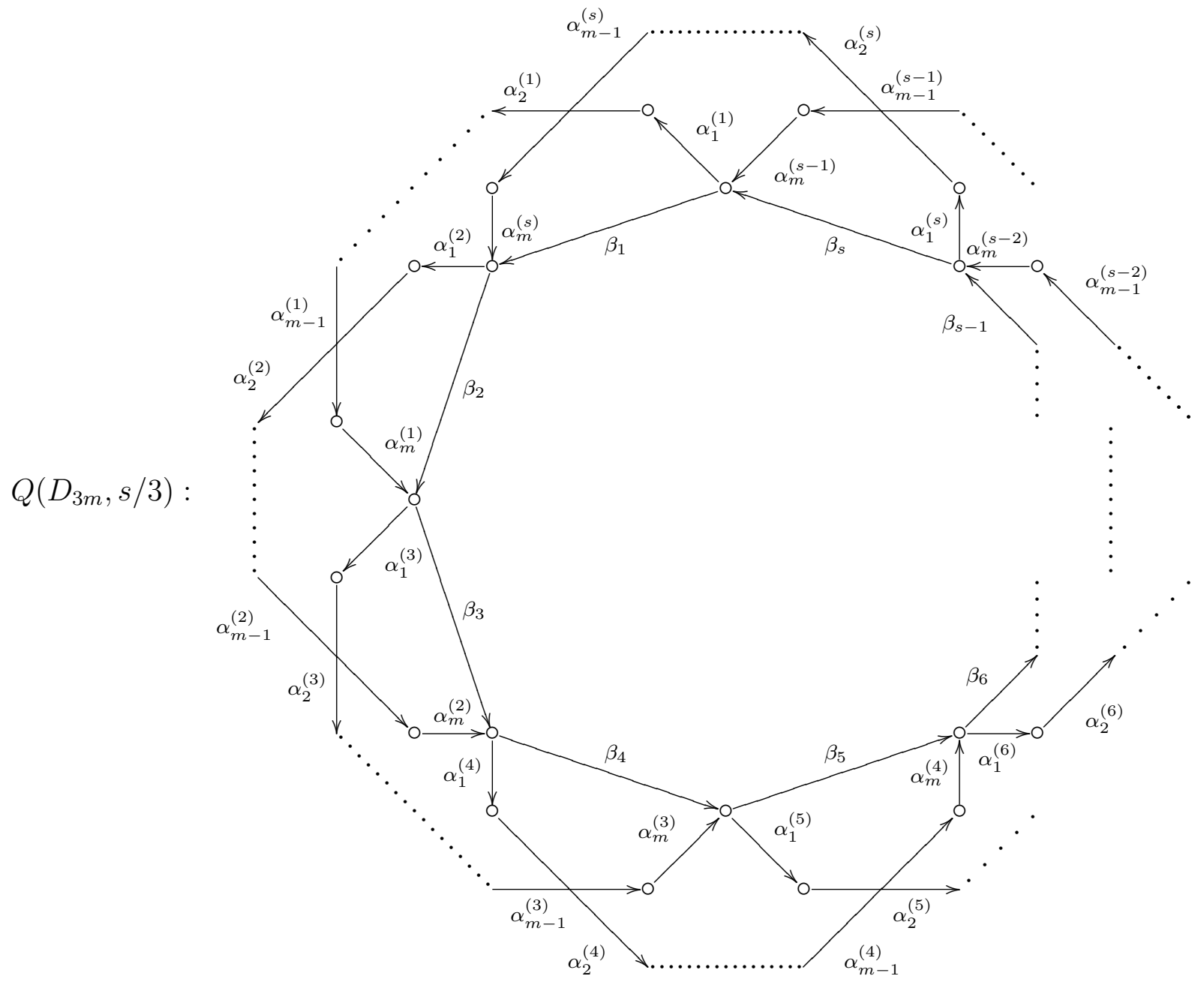

and for $s=1$,

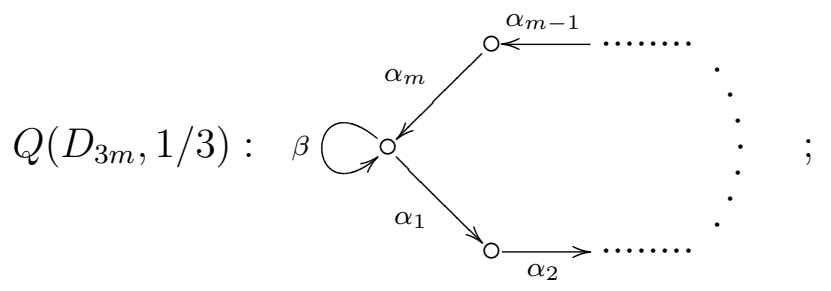

$R\left(D_{3 m}, s / 3\right)$ :

(i) $\alpha_{m}^{(i)} \cdots \alpha_{2}^{(i)} \alpha_{1}^{(i)}=\beta_{i+1} \beta_{i}$ for all $i \in\{1, \ldots, s\}=\mathbb{Z} /\langle s\rangle$;

(ii) $\alpha_{1}^{(i+2)} \alpha_{m}^{(i)}=0$ for all $i \in\{1, \ldots, s\}=\mathbb{Z} /\langle s\rangle$; and

(iii) $\alpha_{j}^{(i+3)} \cdots \alpha_{1}^{(i+3)} \beta_{i+2} \alpha_{m}^{(i)} \cdots \alpha_{j}^{(i)}=0$ for all $i \in\{1, \ldots, s\}=\mathbb{Z} /\langle s\rangle$ and for all $j \in\{1, \ldots, m\}$ (i.e. paths of length $m+2$ are equal to 0 ). 
6.1.7. $\left(E_{n}, s, 1\right)$ with $n \in\{6,7,8\}$ and $s \geq 1 . \Lambda\left(E_{n}, s, 1\right)$ is given by the quiver $Q\left(E_{n}, s\right)$ and relations $R\left(E_{n}, s, 1\right)$ below.

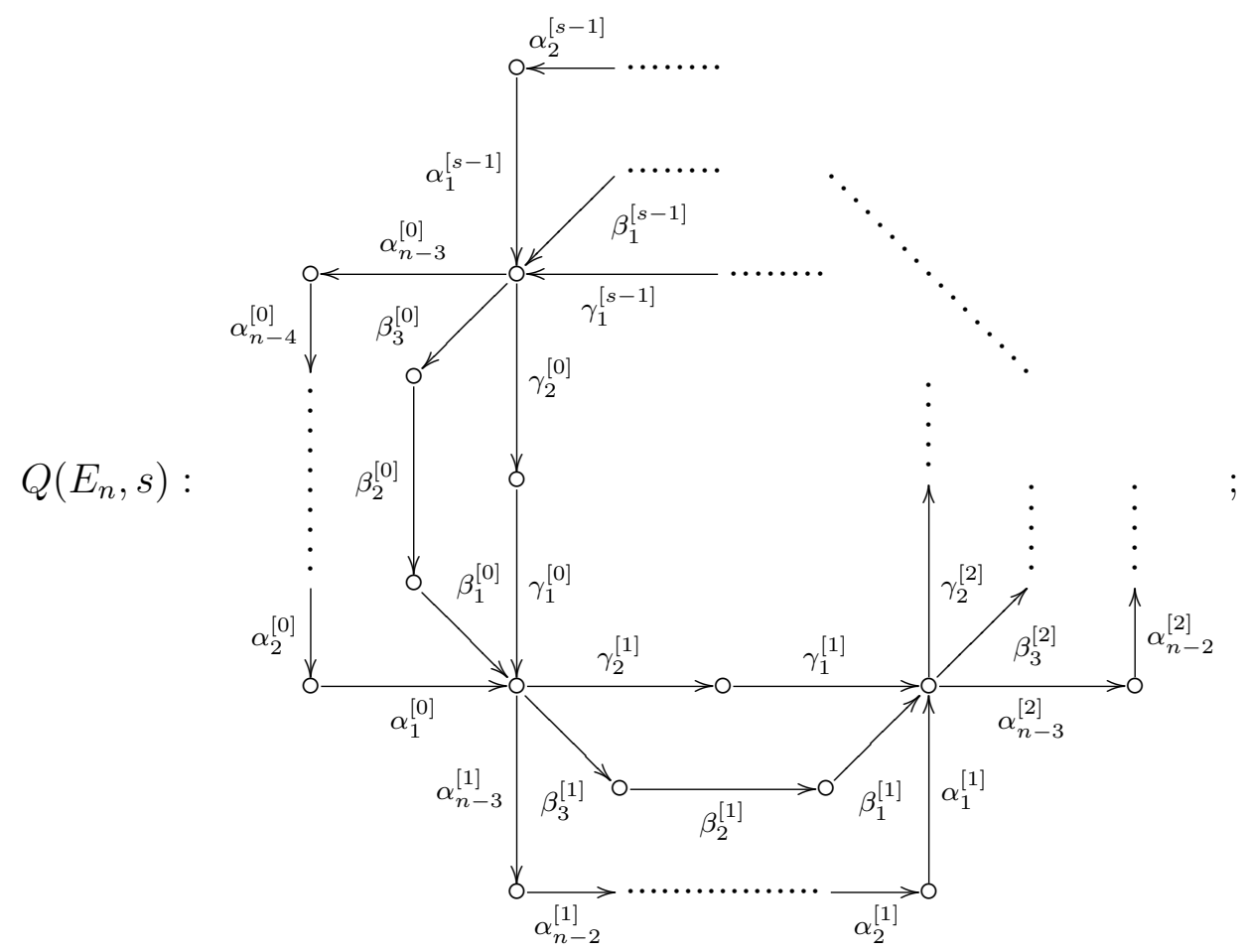

$R\left(E_{n}, s, 1\right)$ :

(i) $\alpha_{1}^{[i]} \alpha_{2}^{[i]} \cdots \alpha_{n-3}^{[i]}=\beta_{1}^{[i]} \beta_{2}^{[i]} \beta_{3}^{[i]}=\gamma_{1}^{[i]} \gamma_{2}^{[i]}$ for all $i \in\{0, \ldots, s-1\}$;

(ii) For all $i \in\{0, \ldots, s-1\}=\mathbb{Z} /\langle s\rangle$,

$$
\left\{\begin{array}{c}
\beta_{3}^{[i+1]} \alpha_{1}^{[i]}=0, \quad \gamma_{2}^{[i+1]} \alpha_{1}^{[i]}=0, \\
\alpha_{n-3}^{[i+1]} \beta_{1}^{[i]}=0, \quad \gamma_{2}^{[i+1]} \beta_{1}^{[i]}=0, \\
\alpha_{n-3}^{[i+1]} \gamma_{1}^{[i]}=0, \quad \beta_{3}^{[i+1]} \gamma_{1}^{[i]}=0 ; \text { and }
\end{array}\right.
$$

(iii) $\alpha$-paths of length $n-2$ are equal to $0, \beta$-paths of length 4 are equal to 0 and $\gamma$-paths of length 3 are equal to 0 .

6.1.8. $\left(E_{6}, s, 2\right)$ with $s \geq 1 . \Lambda\left(E_{6}, s, 2\right)$ is given by the quiver $Q\left(E_{6}, s\right)$ above with relations $R\left(E_{6}, s, 2\right)$ below.

$R\left(E_{6}, s, 2\right)$ :

(i) The same as $R\left(E_{6}, s, 1\right)(\mathrm{i})$;

(ii) For all $i \in\{0, \ldots, s-1\}=\mathbb{Z} /\langle s\rangle$,

$$
\begin{cases}\alpha_{3}^{[i+1]} \gamma_{1}^{[i]}=0, & \beta_{3}^{[i+1]} \gamma_{1}^{[i]}=0 \\ \gamma_{2}^{[i+1]} \alpha_{1}^{[i]}=0, & \gamma_{2}^{[i+1]} \beta_{1}^{[i]}=0\end{cases}
$$


and for all $i \in\{0, \ldots, s-2\}$,

$$
\begin{aligned}
& \beta_{3}^{[i+1]} \alpha_{1}^{[i]}=0, \quad \alpha_{3}^{[i+1]} \beta_{1}^{[i]}=0, \\
& \alpha_{3}^{[0]} \alpha_{1}^{[s-1]}=0, \quad \beta_{3}^{[0]} \beta_{1}^{[s-1]}=0 ; \text { and }
\end{aligned}
$$

(iii) $\gamma$-paths of length 3 are equal to 0 , and for all $i \in\{0, \ldots, s-2\}$ and for all $j \in\{1,2,3\}=\mathbb{Z} /\langle 3\rangle$,

$$
\begin{aligned}
& \alpha_{j-3}^{[i+1]} \cdots \alpha_{j}^{[i]}=0, \quad \beta_{j-3}^{[i+1]} \cdots \beta_{j}^{[i]}=0, \\
& \beta_{j-3}^{[0]} \cdots \beta_{3}^{[0]} \alpha_{1}^{[s-1]} \cdots \alpha_{j}^{[s-1]}=0, \quad \alpha_{j-3}^{[0]} \cdots \alpha_{3}^{[0]} \beta_{1}^{[s-1]} \cdots \beta_{j}^{[s-1]}=0 .
\end{aligned}
$$

6.2. Nonstandard representatives. For each $m \geq 2$ the algebra $\Lambda(m)$ defined by the quiver $Q\left(D_{3 m}, 1 / 3\right)$ above with relations $R(m)$ below is a representation-finite nonstandard selfinjective algebra of type $\left(D_{3 m}, 1 / 3,1\right)$.

$R(m)$ :

(i) $\alpha_{m} \cdots \alpha_{1}=\beta^{2}$;

(ii) $\overbrace{\alpha_{i} \cdots \alpha_{i+1} \alpha_{i}}^{m+1}=0$ for all $i \in\{1, \ldots, m\}=\mathbb{Z} /\langle m\rangle$; and

(iii) $\alpha_{1} \alpha_{m}=\alpha_{1} \beta \alpha_{m}$.

\section{ACKNOWLEDGMENT}

The author would like to thank H. Krause for asking him to give an example of a stable equivalence not of Morita type, from which the present work has started. Most of this work was done while the author visited the University of Bielefeld in 1999/2000. He would like to thank Professor C. M. Ringel for the hospitality, and Sonderforschungsbereich 343 for the support.

\section{REFERENCES}

[1] Asashiba, H.: The derived equivalence classification of representation-finite selfinjective algebras, J. Alg. 214, 1999, 182-221.

[2] Asashiba, H.: On stable equivalences of Morita type between representation-finite selfinjective algebras, The University of Bielefeld, Preprint series of Sonderforschungsbereich 343, Preprint 99-129.

[3] Auslander, M. and Reiten, I.: On a theorem of E. Green on the dual of the transpose, Representations of finite dimensional algebras (Tsukuba, 1990), 53-65, CMS Conf. Proc., 11, Amer. Math. Soc., Providence, RI, 1991.

[4] Bretscher, O., Läser, Ch. and Riedtmann, Ch.: Selfinjective and simply connected algebras, manuscripta math. 36, 1981, 253-307.

[5] Broué, M.: Equivalences of blocks of group algebras, Finite dimensional algebras and related topics, V. Dlab and L. L. Scott (eds.), Kluwer, 1994, 1-26.

[6] Keller, B and Vossieck, D.: Sous les catégories dérivées, C. R. Acad. Sci. Paris, 305, Serie I, 1987, 225-228.

[7] Linckelmann, M.: Stable equivalences of Morita type for self-injective algebras and p-groups, Math. Z. 223, 1996, 87-100.

[8] Rickard, J.: Morita theory for derived categories, J. London Math. Soc., 39 1989, 436-456.

[9] Rickard, J.: Derived categories and stable equivalence, J. Pure and Appl. Alg. 61, 1989, 303-317.

[10] Rickard, J.: Derived equivalences as derived functors, J. London Math. Soc. (2) 43, 1991, 37-48. 
[11] Riedtmann, Chr.: Algebren, Darstellungsköcher, Überlagerungen und zurück, Comm. Math. Helvet. 55, 1980, 199-224.

Department of Mathematics, Osaka City University, 3-3-138 Sugimoto, SumiyoshiKU, OSAKA, 558-8585, JAPAN

E-mail address: asashiba@sci.osaka-cu.ac.jp 